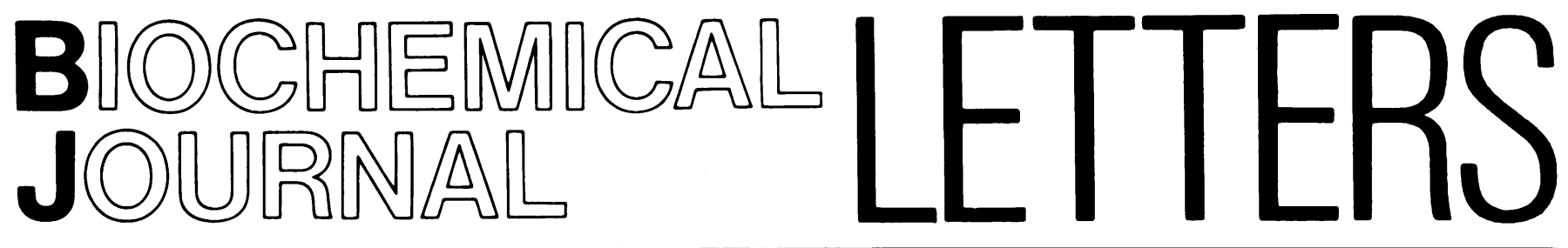

\section{Kinetic parameters of the acyl-enzyme mechanism and conditions for quasi- equilibrium and for optimal catalytic characteristics}

The kinetic parameter $k_{\text {cat. }} / K_{\mathrm{m}}$ (Bender \& Kézdy, 1965; Brocklehurst et al., 1968; Fersht, 1977) is now widely used in the characterization of enzymes and in the study of mechanism. In their discussion of the acyl-enzyme mechanism, eqn. (1), Christensen et al. (1990) suggest that (i) a comparison of steadystate and single-turnover kinetic data can provide a value for $k_{+1}$, the second-order rate constant for the encounter of enzyme and substrate, (ii) the close approximation of $k_{\text {cat. }} / K_{\mathrm{m}}$ and $k_{+2} / K_{\mathrm{s}}$ (i.e. $\left(k_{+1} k_{+2} / k_{-1}\right)$ requires the condition $k_{+1} \gg k_{+2} / K_{\mathrm{s}}$, and (iii) for highly efficiently enzymes that effect catalysis via the acyl-enzyme mechanism, $k_{-1} \simeq k_{+2} \simeq k_{+3}$.

$$
\begin{aligned}
& \mathrm{E}+\mathrm{S} \underset{k_{-1}}{\stackrel{k_{+1}}{\rightleftharpoons}} \mathrm{ES} \stackrel{\boldsymbol{k}_{+2}}{\longrightarrow} \mathrm{ES}^{\prime} \stackrel{\boldsymbol{k}_{+3}}{\longrightarrow} \mathrm{E}+\mathrm{P}_{2} \\
&+ \\
& \mathrm{P}_{1}
\end{aligned}
$$

The purpose of the present letter is to point out that $(a)$ suggestion (i) relies on assumptions about the significance of steady state and single-turnover kinetic data that are mutually incompatible in that if the quasi-equilibrium condition applies to the latter, it necessarily applies also to the former, $(b)$ suggestion (ii) is an unnecessarily restrictive condition for quasi-equilibrium, and (c) suggestion (iii) is not the conclusion deduced from analytical treatment of the acyl-enzyme mechanism (Brocklehurst, 1977).

(a) As Christensen et al. (1990) correctly point out, $K_{\mathrm{m}} / k_{\text {cat. }}$ is related to $K_{\mathrm{s}} / k_{+2}$ by eqn. (2). If an independent estimate of $K_{\mathrm{s}} / k_{+2}$ could be obtained, therefore, for a catalysis where the steady state specificity constant $k_{\text {cat. }} / K_{\mathrm{m}} \neq k_{+2} / K_{\mathrm{s}}$, this would permit calculation of the value of $k_{+1}$. It is well known that for the acyl-enzyme mechanism the meanings of $k_{\text {cat. }}, K_{\mathrm{m}}$ and $k_{\text {cat. }} / K_{\mathrm{m}}$ in terms of rate constants are those given by eqns. (3)-(5) respectively, where $K_{\mathrm{m}(\mathrm{acyl})}$ is the $K_{\mathrm{m}}$ for the overall acylation process.

$$
\begin{gathered}
\frac{K_{\mathrm{m}}}{k_{\text {cat. }}}=\frac{1}{k_{+1}}\left(1+\frac{k_{-1}}{k_{+2}}\right)=\frac{K_{\mathrm{s}}}{k_{+2}}+\frac{1}{k_{+1}} \\
k_{\text {cat. }}=\frac{k_{+2} k_{+3}}{k_{+2}+k_{+3}} \\
K_{\mathrm{m}}=\left(\frac{k_{-1}+k_{+2}}{k_{+1}}\right)\left(\frac{k_{+3}}{k_{+2}+k_{+3}}\right) \\
\frac{k_{\text {cat. }}}{K_{\mathrm{m}}}=\frac{k_{+1} k_{+2}}{k_{-1}+k_{+2}}=\frac{k_{+2}}{K_{\mathrm{m}(\mathrm{acyl})}}
\end{gathered}
$$

Analysis of the second transient phase of the progress curve for the production of $\mathrm{P}_{1}$ with $[\mathrm{S}]_{0} \gg[\mathrm{E}]_{\mathrm{T}}$ or of the production of $\mathrm{P}_{1}$ in a single-turnover reaction with $[\mathrm{E}]_{\mathrm{T}} \gg[\mathrm{S}]_{0}$ can provide values of $k_{+2}$ and $K_{\text {m(acyl) }}$ (see e.g. Gutfreund \& Sturtevant, 1956; Gutfreund, 1965; Wharton \& Eisenthal, 1981). The reason that it is not possible to use eqn. (2) in conjunction with steady state and single-turnover or pre-steady-state data (i.e. data obtained either with $[\mathrm{E}]_{\mathrm{T}} \gg[\mathrm{S}]_{0}$, or with $[\mathrm{S}]_{0} \gg[\mathrm{E}]_{\mathrm{T}}$ when $k_{+3} \ll k_{+2}$ and $\simeq$ zero) to obtain a value for $k_{+1}$ is that for a given catalysis both methods of kinetic analysis provide the same quantity, $k_{+2} K_{\mathrm{m}(\mathrm{acyl})}$, whether or not this happens to approximate closely to $k_{+2} / K_{\mathrm{s}}$. Although in principle it is possible to obtain values of $k_{+1}$ and $k_{-1}$ from the information contained in the first transient phase, i.e. the lag phase during pre-steady-state acceleration of enzyme reactions (see e.g. Gutfreund, 1965; Wharton \& Eisenthal, 1981), in practice, even with precise measurements, it is difficult or impossible to determine the observed rate constant and the associated amplitude factor of the first transient phase by using readily available equipment such as stopped-flow systems.

(b) Consideration of eqn. (2) does lead to the conclusion that $k_{\text {cat. }} / K_{\mathrm{m}} \simeq k_{+2} / K_{\mathrm{s}}$ if $k_{+2} / K_{\mathrm{s}} \ll k_{+1}$, but this is not a necessary condition for quasi-equilibrium. The somewhat less onerous condition $k_{\text {cat. }} / K_{\mathrm{m}} \ll k_{+1}$ (i.e. $k_{+2} / K_{\mathrm{m}(\mathrm{acyl})} \ll k_{+1}$ ) also provides that $K_{\mathrm{m}} \simeq K_{\mathrm{s}}$ [see Brocklehurst, 1979, and note that eqn. (2) of that paper which was incorrectly printed as $k=k_{+1} k_{+2} / k_{-1} k_{+2}$ should read $k=k_{+1} k_{+2} /\left(k_{-1}+k_{+2}\right)$ ]. Thus eqn. (5) may be transformed into eqn. (6), where $k \equiv k_{\text {cat. }} / K_{\mathrm{m}}$ (or alternatively the second-order rate constant for reaction of enzyme with a timedependent inhibitor), from which it is clear that the conventional condition for quasi-equilibrium, $k_{+2} \ll k_{-1}$, is a necessary consequence of the condition $k \ll k_{+1}$.

$$
\frac{k_{+2}}{k_{-1}}=\frac{k / k_{+1}}{1-\left(k / k_{+1}\right)}
$$

(c) The evolutionary optimization of catalytic effectiveness continues to attract considerable interest (see e.g. Burbaum et al., 1989; Burbaum \& Knowles, 1989; Petterssen, 1989). The effectiveness of enzyme catalysis has been treated analytically (Brocklehurst, 1977) both in terms of what might be regarded as the simplest plausible kinetic model [eqn. (7)] (Haldane, 1930) and in terms of acyl-enzyme mechanism [eqn. (1)] (Hartley \& Kilby, 1952, 1954) as a restricted version of eqn. (7).

$$
\mathrm{E}+\mathrm{S} \underset{k_{-1}}{\stackrel{k_{+1}}{\rightleftharpoons}} \mathrm{ES} \underset{k_{-2}}{\stackrel{k_{+2}}{\rightleftharpoons}} \mathrm{EP} \underset{k_{-3}}{\stackrel{k_{+3}}{\rightleftharpoons}} \mathrm{E}+\mathrm{P}
$$

To assess the effectiveness of an enzyme as a catalyst for the conversion of $\mathbf{S}$ into $\mathbf{P}$, it is necessary to determine the extent to which the constraints of a particular kinetic mechanism will permit $v_{2}$ [the rate when $K_{\mathrm{m}} \gg[\mathrm{S}]$, eqn. (8)] to approach the diffusion-limited rate, $v_{\mathrm{d}}$ [eqn. (9)].

$$
\begin{aligned}
& v_{2}=\frac{k_{\text {cat. }}}{K_{\mathrm{m}}}[\mathrm{E}][\mathrm{S}] \\
& v_{\mathrm{d}}=k_{+1}[\mathrm{E}][\mathrm{S}]
\end{aligned}
$$


The theoretical limit on $v_{2}$ (in terms of $v_{\mathrm{d}}$ ) is dictated by the value of the equilibrium constant for the conversion of $\mathrm{S}$ into $\mathrm{P}\left(K_{\mathrm{eq}}\right)$. The optimal rate of catalysis, $v_{\text {opt. }}$ (the theoretical limiting value of $v_{2}$ ) may take values between $v_{\mathrm{d}}$, when $K_{\mathrm{eq} .} \gg 1$, and $K_{\mathrm{eq}} \cdot v_{\mathrm{d}}$, when $K_{\mathrm{eq}} \ll 1$. Analysis (Brocklehurst, 1977) of the Haldane (1930) model [eqn. (7)] shows that optimal catalysis requires that $k_{+2} \gg k_{-1}$ unless $K_{\text {eq. }} \ll 1$. If $K_{\text {eq. }} \ll 1$, it is necessary only that $k_{+2} \gg K_{\text {eq. }} \cdot k_{-1}$, which means that there can be optimal catalysis even if $k_{+2}<k_{-1}$. For reactions with $K_{\text {eq. }}=1$ and $k_{+2}=k_{-2}$, $v_{2}=v_{\text {opt. }}$ if $k_{+2} \gg k_{-1}$ (and necessarily, therefore, $k_{+3} \ll k_{-2}$ ). The value of $v_{\text {opt. }}$, however, is not $v_{\mathrm{d}}$ but only $50 \%$ of this value. The energy barrier for ES $\rightarrow$ EP is below those for the other two steps, which are the only two that contribute significantly to rate limitation, and hence $v_{\text {opt. }}=v_{\mathrm{d}} / 2$. For reactions with $K_{\text {eq. }} \gg 1$, which include those that obey the acyl-enzyme mechanism of Hartley \& Kilby $(1952,1954)$ [eqn. (1)], $v_{\text {opt. }}=v_{\mathrm{d}}$. The fact that $K_{\text {eq }} \gg 1$ means that the energy barriers for ES $\rightarrow$ EP and for $E P \rightarrow E+P$ can be below that for $E+S \rightarrow E S$, which can, therefore, become rate-limiting. If substrate binding and product binding are equally strong, the necessity that $k_{+2} \gg k_{-1}$ for optimal catalysis implies that $k_{+2} \gg k_{+3}$. In other cases, $v_{2}=v_{\mathrm{d}}=v_{\text {opt. }}$, whatever the relative values of $k_{+2}$ and $k_{+3}$, provided that $k_{+2} \gg k_{-1}$. The demand that $k_{+2} \gg k_{-1}$ for the hypothetical optimal enzyme (for a reaction in which $K_{\text {eq. }} \gg 1$ ) might be modulated to $k_{+2} \simeq k_{-1}$ by the requirement for even partitioning of intermediates discussed by Knowles (see e.g. Knowles, 1976). The condition $k_{+2}=k_{-1}$ alone represents quite a small sacrifice in effectiveness of catalysis: when $K_{\text {eq. }}>1, v_{2}$ is decreased from $v_{\text {opt. }}=v_{\mathrm{d}}$ to $v_{\mathrm{d}} / 2$, and, when $K_{\text {eq. }}=1, v_{2}$ is decreased from $v_{\text {opt. }}=v_{\mathrm{d}} / 2$ to $v_{\mathrm{d}} / 3$.

There are clearly circumstances in kinetic analysis where it is important to distinguish between $K_{\mathrm{s}}$ and more complex assemblies of rate constants such as $K_{\mathrm{m}(\mathrm{acyl})}$ and to properly establish the conditions that define the state of quasi-equilibrium (see e.g. Brocklehurst \& Cornish-Bowden, 1976; Brocklehurst \& Dixon, 1976, 1977). There is an unfortunate tendency in some papers in the literature to make the unstated assumption of quasi-equilibrium and to write $K_{\mathrm{s}}$ instead of $\left(k_{-1}+k_{+2}\right) / k_{+1}$ in what can appear to others to be kinetic statements of general applicability (for a recent example, see Aducci et al., 1988, but cf. Ascenzi et al., 1989, where conditions for quasi-equilibrium are explicitly stated for both the pre-steady state and the steady state). Sometimes this does not affect the analysis of the particular problem that is being addressed, but sometimes it does!

Another example of a paper in which $K_{\mathrm{s}}$ is written instead of $\left(k_{-1}+k_{+2}\right) /\left(k_{+1}\right)$ [i.e. instead of $K_{\mathrm{m}(\mathrm{acyl})}$, see eqn. (5)] is that by Pratt et al. (1988) on the reaction of Staphylococcus aureus PC1 $\beta$ lactamase with dansylpenillin. Christensen et al. (1990) followed Pratt et al. (1988) in assuming that the stopped-flow kinetic data provided values of $k_{+2} / K_{\mathrm{s}}$ rather than $k_{+2} / K_{\mathrm{m}(\mathbf{a c y l})}$. They then used eqn. (2) of the present letter in an attempt to explain the apparent discrepancy between the value of $k_{+2} / K_{\mathrm{m}(a c y l)}$ obtained from the stopped-flow data $\left(2.5 \times 10^{6} \mathrm{M}^{-1} \cdot \mathrm{s}^{-1}\right)$ and the value of $k_{\text {cat. }} / K_{\mathrm{m}}$ obtained from steady state kinetic experiments $\left(1.1 \times 10^{6} \mathrm{M}^{-1} \cdot \mathrm{s}^{-1}\right)$. As pointed out above, use of eqn. (2) in this way is inappropriate. A possible and trivial explanation for the apparent discrepancy may be the uncertainty of the values of the parameters obtained from the stopped-flow experiments which were carried out with [S] $<70 \mu \mathrm{M}$ even though the value of $K_{\text {m(acyl) }}$ [written by Pratt et al. (1988) as $K_{\mathrm{s}}$ ] was determined as $70 \pm 30 \mu \mathrm{M}$, an uncertainty pointed out by Pratt et al. (1988).

The other difference between the results of the kinetic experiments on $\beta$-lactamases reported by Christensen et al. (1990) and those reported by Pratt et al. (1988) is that the former suggest that $k_{+2} \simeq k_{+3}$ (at least at $\mathrm{pH}$ 7) whereas the latter report that deacylation is rate-limiting in the hydrolysis of good substrates (at least at pH 9). Christensen et al. (1990) studied the hydrolysis of benzylpenicillin catalysed by three $\beta$-lactamases and determined values of $k_{+2}$ and $k_{+3}$ by acid-quench experiments and values of $k_{+1}$ and $k_{-1}$ from experiments in which the viscosity of the solvent was varied by inclusion of sucrose or glycerol. For the reaction catalysed by $\beta$-lactamase $\mathrm{I}, k_{\text {cat. }} / K_{\mathrm{m}}=2.6 \times 10^{7}$ $\mathrm{M}^{-1} \cdot \mathrm{s}^{-1}$ and $k_{+1}=4.1 \times 10^{7} \mathrm{M}^{-1} \cdot \mathrm{s}^{-1}$, both of which are close to the lower end of the range of values predicted for a diffusionlimited enzyme reaction $\left(1 \times 10^{8}-1 \times 10^{10} \mathrm{M}^{-1} \cdot \mathrm{s}^{-1}\right.$; see Blacklow et al., 1988, for a discussion), $k_{-1}=2.3 \times 10^{3} \mathrm{~s}^{-1}$, and $k_{+2}=$ $4.1 \times 10^{3}$. In terms of the analytical treatment of enzyme effectiveness (Brocklehurst, 1977) discussed above, these values (with $k_{+2}>k_{-1}$ ) support the suggestion by Christensen et al. (1990) that $\beta$-lactamase $I$ is a highly efficient enzyme. It is important to emphasize that this conclusion is valid whatever the relative values of $k_{+2}$ and $k_{+3}$ and so the unresolved difference between the results of Christensen et al. (1990) and those of Pratt et al. (1988) does not affect the conclusion about enzyme efficiency. The same conclusion does not apply to the same extent to the reaction catalysed by RTEM $\beta$-lactamase. For this catalysis the values of the rate constants reported by Christensen et al. (1990) are $k_{\text {cat. }} / K_{\mathrm{m}}=2.4 \times 10^{7} \mathrm{M}^{-1} \cdot \mathrm{s}^{-1}, k_{+1}=1.23 \times 10^{8} \mathrm{M}^{-1} \cdot \mathrm{s}^{-1}, k_{-1}=$ $1.2 \times 10^{4} \mathrm{~s}^{-1}$, and $k_{+2}=2.8 \times 10^{3} \mathrm{~s}^{-1}$. The fact that the value of $k_{+2}$ is substantially smaller than the value of $k_{-1}$ is diagnostic of a less efficient enzyme.

We thank the Science and Engineering Research Council for project grants and Earmarked Research Studentships for work involving kinetic study of enzyme reactions.

\section{Keith BROCKLEHURST ${ }^{*} \ddagger$ and Christopher M. TOPHAM $\dagger$}

*Department of Biochemistry, Medical College of St. Bartholomew's Hospital, University of London, Charterhouse Square, London EC1M 6BQ, U.K., and †Laboratory of Molecular Biology, Department of Crystallography, Birkbeck College, University of London, Malet Street, London WCIE 7HX, U.K.

¥Present address: Department of Biochemistry, Queen Mary and Westfield College, University of London, Mile End Road, London E1 4NS, U.K

Aducci, P., Ascenzi, P., Amiconi, G. \& Galbio, A. (1988) J. Mol. Catalysis 47, 343-350

Ascenzi, P., Menegatti, E., Guarneri, M. \& Amicone, G. (1989) Biochim. Biophys. Acta 998, 210-214

Bender, M. L. \& Kézdy, F. J. (1965) Annu. Rev. Biochem. 34, 49-76

Blacklow, S. C., Raines, R. T., Lim, W. A., Zamore, P. D. \& Knowles, J. R. (1988) Biochemistry 27, 1158-1167

Brocklehurst, K. (1977) Biochem. J. 163, 111-116

Brocklehurst, K. (1979) Biochem. J. 181, 775-778

Brocklehurst, K. \& Cornish-Bowden, A. (1976) Biochem. J. 159, 165-166

Brocklehurst, K. \& Dixon, H. B. F. (1976) Biochem. J. 155, 61-70

Brocklehurst, K. \& Dixon, H. B. F. (1977) Biochem. J. 167, 859-862

Brocklehurst, K., Crook, E. M. \& Wharton, C. W. (1968) FEBS Lett. 2, 69-73

Burbaum, J. J. \& Knowles, J. R. (1989) Biochemistry 28, 9306-9317

Burbaum, J. J., Raines, R. T., Albery, W. J. \& Knowles, J. R. (1989) Biochemistry 28, 9293-9305

Christensen, H., Martin, M. T. \& Waley, S. G. (1990) Biochem. J. 266, 853-861

Fersht, A. (1977) Enzyme Structure and Mechanism, pp. 96-97, W. H. Freeman \& Co., Reading.

Gutfreund, H. (1965) An Introduction to the Study of Enzymes, pp. 50-61, John Wiley \& Sons, London

Gutfreund, H. \& Sturtevant, J. M. (1956) Biochem. J. 63, 656-661

Haldane, J. B. (1930) Enzymes, Longmans, London

Hartley, B. S. \& Kilby, B. A. (1952) Biochem. J. 50, 672-678

Hartley, B. S. \& Kilby, B. A. (1954) Biochem. J. 56, 288-297

Knowles, J. R. (1976) FEBS Lett. 62 (Suppl.) E53-E61

Petterssen, G. (1989) Eur. J. Biochem. 184, 561-566 
Pratt, R. F., McConnell, T. S. \& Murphy, S. J. (1988) Biochem. J. 254, 916-922

Wharton, C. W. \& Eisenthal, R. (1981) Molecular Enzymology, pp. 204-214, Blackie \& Son, Glasgow

Received 26 March 1990

\section{Kinetic parameters of the acyl-enzyme mechanism}

The preceding letter (Brocklehurst \& Topham, 1990) comments on three points made by Christensen et al. (1990): two concern kinetic parameters and quasi-equilibrium in the acyl-enzyme mechanism and the third concerns the expected relationships between the rate constants for a fully efficient enzyme.

Their comments on this last point are discussed first. Our conclusions did indeed differ from those of Brocklehurst (1977); this was because we were interpreting our results according to Burbaum et al. (1989). For the acyl-enzyme mechanism [eqn. (1) of the preceding letter] Brocklehurst (1977) states that, unless substrate binding and product binding are equally strong, $k_{+2} \gg k_{-1} ; k_{+2} / k_{+3}$ is not specified. On the other hand, Burbaum et al. (1989) infer that $k_{+2} \cong k_{+3}$, and that at (or near to) the diffusion limit the barrier for the second transition state is likely to be as low as it needs to be to avoid slowing the reaction, and so $k_{-1} \cong k_{+2}$. Brocklehurst (1977) did suggest that the condition $k_{+2} \gg k_{-1}$ might be relaxed to $k_{+2} \cong k_{-1}$ with little loss in catalytic efficiency, and so this leaves the main difference between Brocklehurst (1977) and Burbaum et al. (1989) as the additional requirement that $k_{+2} \cong k_{+3}$ in the latter treatment. Our experimental results for $\beta$-lactamases (Christensen et al., 1990) were that $k_{-1} \cong k_{+2} \cong k_{+3}$, and that the reactions were partly (or largely) diffusion-controlled; thus our results were entirely consistent with the theories of Burbaum et al. (1989).

We come now to the significance of the parameters obtained by steady-state and single-turnover kinetics. Single-turnover kinetics, applicable when the concentration of enzyme is much greater than that of the substrate, give an equation for $P_{1}$ in the acyl-enzyme mechanism [eqn. (1) of the preceding letter] that has two exponential terms (see, e.g., Kasserra \& Laidler, 1970). The useful (and usual) approximate treatment of single-turnover kinetics for the acyl-enzyme mechanism (which neglects the first transient) does indeed give $\left(k_{-1}+k_{+2}\right) / k_{+1}$, the parameter referred to as $K_{\mathrm{m} \text { (acyl) }}$ by Brocklehurst \& Topham (1990), $K^{\prime}$ by Christensen et al. (1990) and $K^{*}$ by Kasserra \& Laidler. The approximation can be tested when all the rate constants are known. For example, if $k_{+1}, k_{-1}$ and $k_{+2}$ are $22 \mu \mathrm{M}^{-1} \cdot \mathrm{s}^{1}, 196 \mathrm{~s}^{-1}$ and $173 \mathrm{~s}^{-1}$ respectively [the values from Christensen et al. (1990) for $\beta$ lactamase PC1], and the concentration of enzyme is $15 \mu \mathrm{M}$, then the approximate treatment would give a value for $k_{+2}$ (given $K^{\prime}$ ) that is about $10 \%$ low; the first transient would be not normally be observed in practice. The concentration of substrate is assumed to be negligible compared with that of the enzyme in such treatments; in practice this is often inconvenient, and may be circumvented by the extrapolation procedure of Crompton \& Waley (1989) \{in that paper, the equation for $y+p$ should read $s\left[1-\exp \left(-\lambda_{2} \cdot t\right)\right]$ not $s\left[-\exp \left(-\lambda_{2} \cdot t\right)\right]$ and the equation for $\lambda_{2}$ should read $k_{+1}^{\prime} \cdot k_{2} /\left(k_{-1}+k_{+1}^{\prime}+k_{+2}\right)$ not $\left.k_{+1}^{\prime} \cdot k_{+2} /\left(k_{-1}+k_{+1}^{\prime}\right)\right\}$.

It is clear from eqn. (2) of the preceding letter that $K_{\mathrm{m}} / k_{\text {cat. }}=\left(k_{-1}+k_{+2}\right) /\left(k_{+1} k_{+2}\right)=K^{\prime} / k_{+2} ;$ both single-turnover and steady-state treatments do indeed give $K^{\prime}$ as correctly pointed out by Brocklehurst \& Topham (1990), and the method of determining the association rate $\left(k_{+1}\right)$ in the Appendix of Christensen et al. (1990) is mistaken. However, to come to practicalities, a useful way to determine $k_{+1}$ is from the dependence of $K_{\mathrm{m}} / k_{\text {cat. }}$ on viscosity (Hardy \& Kirsch, 1984; Blacklow et al., 1988; Christensen et al., 1990); moreover this procedure also gives $k_{-1} / k_{+2}$ and so shows directly whether the quasi-equilibrium assumption is valid. Thus the comparison of $k_{\text {cat. }} / K_{\mathrm{m}}$ and $k_{+1}$ which Brocklehurst \& Topham (1990) commend as a test for quasi-equilibrium is unneeded if the viscosity method is used to find $k_{+1}$.

\section{Stephen G. WALEY}

Laboratory of Molecular Biophysics, University of Oxford, Rex Richards Building, South Parks Road, Oxford, OX1 3QU, U.K., and Oxford Centre for Molecular Sciences, Oxford, U.K.

Blacklow, S. C., Raines, R. T., Lim, W. A., Zamore, P. D. \& Knowles, J. R. (1988) Biochemistry 27, 1158-1167

Brocklehurst, K. (1977) Biochem. J. 163, 111-116

Brocklehurst, K. \& Topham, C. M. (1990) Biochem. J. 270, 561-562

Burbaum, J. J., Raines, R. T., Albery, W. J. \& Knowles, J. R. (1989) Biochemistry 28, 9293-9305

Christensen, H., Martin, M. T. \& Waley, S. G. (1990) Biochem. J. 266, 853-861; see also erratum in Biochem. J. (1990) 268, 808

Crompton, I. E. \& Waley, S. G. (1989) Biochem. J. 257, 305-308

Hardy, L. W. \& Kirsch, J. F. (1984) Biochemistry 23, 1275-1282

Kasserra, H. P. \& Laidler, K. J. (1970) Can. J. Chem. 48, 1793-1802

Received 4 June 1990

\section{Questions about the use of $\left[{ }^{3} \mathrm{H}\right]$ thymidine incorporation as a reliable method to estimate cell proliferation rate}

Control of cell proliferation is raising interest in an increasing number of research centres, appearing as it does to be one of the most promising fields of research. It involves studies on rapidly expanding subjects such as growth factors, hormones, transmembrane signalling, kinases, second messengers, ornithine decarboxylase, oncogenes, etc. It constitutes a basic approach to important questions such as embryogenesis, wound healing, cancer, etc. From a survey of the related recent literature, it appears that a large part, if not most, of the research teams use incorporation of radiolabelled thymidine (mostly ${ }^{3} \mathrm{H}$-labelled) into DNA as an index of cell proliferation. In many cases, number of c.p.m. after exposure to $\left[{ }^{3} \mathrm{H}\right]$ thymidine is reported as the sole index of cell growth.

Theoretically, the method is very attractive because it relies on a very simple principle: labelled thymidine added to the culture medium will be incorporated into DNA during the $S$ phase of the cell cycle. As the rate of DNA synthesis expresses the mitotic activity, the radioactivity of trichloroacetic acid-precipitable material should reflect cell growth. Such a method is both easier to carry out and less time-consuming than direct cell counting under the microscope, an alternative to estimate cell growth. Besides, its wide use gives the feeling that there are few questions about its reliability.

In practice, authors use either the pulse labelling method or the continuous labelling method. Surprisingly, detailed protocols described in published papers rarely refer to first or previous authors having described one method or the other. It underscores the fact that there are multiple adaptations and variations of the protocols. It seems that the pulse method was described as early as 1953 [1]. Detailed descriptions of both methods and pertinent criticism of them can be found in a book published in 1980 [2].

Starting a study on the ability of some external signals to control proliferation of HT29 human colon cancer cells in 